(c) Inra/Elsevier, Paris

Original article

\title{
Blocking Gibbs sampling in the mixed inheritance model using graph theory
}

\author{
Mogens Sandø Lund ${ }^{\mathrm{a} *}$, Claus Skaanning Jensen ${ }^{\mathrm{b}}$, \\ ${ }^{a}$ DIAS, Department of Breeding and Genetics, Research Centre Foulum, \\ P.O. Box 50, 8830 Tjele, Denmark \\ ${ }^{b}$ AUC, Department of Computer Science, Fredrik Bajers Vej 7E., \\ 9220 Aalborg $\varnothing$, Denmark
}

(Received 10 February 1998; accepted 18 November 1998)

\begin{abstract}
For the mixed inheritance model (MIM), including both a single locus and a polygenic effect, we present a Markov chain Monte Carlo (MCMC) algorithm in which discrete genotypes of the single locus are sampled in large blocks from their joint conditional distribution. This requires exact calculation of the joint distribution of a given block, which can be very complicated. Calculations of the joint distributions were obtained using graph theoretic methods for Bayesian networks. An example of a simulated pedigree suggests that this algorithm is more efficient than algorithms with univariate updating or algorithms using blocking of sires with their final offspring. The algorithm can be extended to models utilising genetic marker information, in which case it holds the potential to solve the critical reducibility problem of MCMC methods often associated with such models. (C) Inra/Elsevier, Paris
\end{abstract}

blocking / Gibbs sampling / mixed inheritance model / graph theory / Bayesian network

Résumé - Échantillonnage de Gibbs par bloc dans le modèle à hérédité mixte en utilisant la théorie des graphes. Pour le cas de l'hérédité mixte (un seul locus avec un fond polygénique), on présente un algorithme de Monte-Carlo par chaînes de Markov (MCMC) dans lequel les génotypes au locus unique sont échantillonnés en blocs importants à partir de leur distribution jointe conditionnelle. Ceci exige le calcul exact de distribution conjointe d'un bloc donné qui peut être très compliquée. Le calcul des distributions jointes est obtenu en utilisant des méthodes graphiques théoriques pour les réseaux bayésiens. Un exemple de pedigree simulé suggère que cet algorithme est plus efficace que les algorithmes à mise à jour univariants ou par groupes de descendance issue de même père. Cet algorithme peut être étendu à des

* Correspondence and reprints
E-mail: mogens.lund@agrsci.dk 
modèles utilisant l'information de marqueurs génétiques ce qui permet d'éliminer le risque de réductibilité souvent associé à de tels modèles quand on applique des méthodes MCMC. (C) Inra/Elsevier, Paris

blocage / échantillonnage de Gibbs / modèle à hérédité mixte / théorie des graphes / réseau bayésien

\section{INTRODUCTION}

In mixed inheritance models (MIM), it is assumed that phenotypes are influenced by the genotypes at a single locus and a polygenic component [19]. Unfortunately, it is not feasible to maximise the likelihood function associated with such models using analytical techniques. Even in the case of single gene models without polygenic effects, the need to marginalise over the distribution of the unknown single genotypes results in computations which are not feasible. For this reason, Sheehan [20] used the local independence structure of genotypes to derive a Gibbs sampling algorithm for a one-locus model. This technique circumvented the need for exact calculations in complex joint genotypic distributions as the Gibbs sampler only requires knowledge of the full conditional distributions.

Algorithms for the more complex MIMs were later implemented using either a Monte Carlo EM algorithm [8], or a fully Bayesian approach [9] with the Gibbs sampler. However, Janss et al. [9] found that the Gibbs sampler had very poor mixing properties owing to a strong dependency between genotypes of related individuals. They also noticed that the sample space was effectively partitioned into subspaces between which movement occurred with low probability. This occurred because some discrete genotypes rarely changed states. This is known as practical reducibility. Both the mixing and reducibility properties are vastly improved by sampling genotypes jointly. Consequently, Janss et al. [9] applied a blocking strategy with the Gibbs sampler, in which genotypes of sires and their final offspring (non-parents), were sampled simultaneously from their joint distribution (sire blocking). This blocking strategy made it simple to obtain exact calculations of the joint distribution and improved the mixing properties in data structures with many final offspring. However, the blocking strategy of Janss and co-workers is not a general solution to the problem because final offspring may constitute only a small fraction of all individuals in a pedigree.

An extension of another blocking Gibbs sampler developed by Jensen et al. [13] could provide a general solution to MIMs. Their sampler was for onelocus models, and sampled genotypes of many individuals jointly, even when the pedigree was complex. The method relied on a graphical model representation and treated genotypes as variables in a Bayesian network. This results in a graphical representation of the joint probability distribution for which efficient algorithms to perform exact inference exist (e.g. [16]). However, a constraint of the blocking Gibbs sampler developed by Jensen and co-workers is that it only handles discrete variables, and in turn cannot be used in MIMs.

The objective of this study is to extend the blocking Gibbs sampler of Jensen et al. [13] such that it can be used in MIMs. A simulated example is presented to illustrate the practicality of the proposed method. The data from the example were also analysed by the method proposed by Janss et al. [9], for comparison. 


\section{MATERIALS AND METHODS}

\subsection{Mixed inheritance model}

In the MIM, phenotypes are assumed to be influenced by the genotype at a single major locus and a polygenic effect. The polygenic effect is the combined effect of many additive and unlinked loci, each with a small effect. Classification effects (e.g. herd, year or other covariates) can easily be included in the model.

The statistical model for a MIM is defined as:

$$
\mathbf{y}=\mathbf{X b}+\mathbf{Z u}+\mathbf{Z W} \mathbf{m}+\mathbf{e}
$$

where $\mathbf{y}$ is a $\left(n^{*} 1\right)$ vector of $n$ observations, $\mathbf{b}$ is a $\left(p^{*} 1\right)$ vector of $p$ classification effects, $\mathbf{u}$ is a $\left(q^{*} 1\right)$ vector of $q$ random polygenic effects, $\mathbf{m}$ is a $\left(3^{*} 1\right)$ vector of genotype effects and $\mathbf{e}$ is a $\left(n^{*} 1\right)$ vector of $n$ random residuals. $\mathbf{X}$ is a $\left(n^{*} r\right)$ design matrix associating data with the 'fixed' effects, and $\mathbf{Z}$ a $\left(n^{*} q\right)$ design matrix associating data with polygenic and single gene effects. $\mathbf{W}$ is an unknown $\left(q^{*} 3\right)$ random design matrix of genotypes at the single locus.

Given location and scale parameters, the data are assumed to be normally distributed as

$$
\mathbf{y} \mid \mathbf{b}, \mathbf{u}, \mathbf{W} \mathbf{m}, \sigma_{\mathrm{e}}^{2} \sim \mathrm{N}\left(\mathbf{X} \mathbf{b}+\mathbf{Z u}+\mathbf{Z W} \mathbf{m}, \mathbf{I} \sigma_{\mathrm{e}}^{2}\right)
$$

where $\sigma_{\mathrm{e}}^{2}$ is the residual variance. For polygenic effects, we invoke the infinitesimal additive genetic model [1], resulting in normally distributed polygenic effects, such that

$$
\mathbf{u} \mid \mathbf{A}, \sigma_{\mathrm{u}}^{2} \sim \mathrm{N}\left(\mathbf{0}, \mathbf{A} \sigma_{\mathrm{u}}^{2}\right)
$$

where $\mathbf{A}$ is the known additive relationship matrix describing the family relations between individuals, and $\sigma_{u}^{2}$ is the additive variance of polygenic effects.

The single locus was assumed to have two alleles $\left(A_{1}\right.$ and $\left.A_{2}\right)$, such that each individual had one of the three possible genotypes: $A_{1} A_{1}, A_{1} A_{2}$ and $A_{2} A_{2}$. For each individual in the pedigree, these genotypes were represented as a random vector, $\mathbf{w}_{\mathrm{i}}$, taking values $(100),\left(\begin{array}{lll}0 & 10\end{array}\right)$ or $\left(\begin{array}{ll}0 & 01)\end{array}\right)$. The vectors $\mathbf{w}_{\mathrm{i}}$ form the rows of $\mathbf{W}$ and will for notational convenience be referred to as $\omega_{1}, \omega_{2}$ and $\omega_{3}$. For individuals which do not have known parents (i.e. founder individuals) the probability distribution of genotype $\mathbf{w}_{\mathrm{i}}$ was assumed to be $p\left(\mathbf{w}_{\mathrm{i}} \mid f\right)$. The distribution for genotype frequency of the base population $(f)$, was assumed to follow Hardy-Weinberg proportions. For individuals with known parents, the genotype distribution is denoted as $p\left(\mathbf{w}_{\mathbf{i}} \mid \mathbf{w}_{\text {sire(i })}, \mathbf{w}_{\text {dam(i) }}\right)$. This distribution describes the probability of alleles constituting genotype $\mathbf{w}_{\mathrm{i}}$, being transmitted from parents with genotypes $\mathbf{w}_{\text {sire(i) }}$ and $\mathbf{w}_{\text {dam(i) }}$ when segregation of alleles follows Mendelian transmission probabilities. For individuals with only one known parent, a dummy individual is inserted for the missing parent.

Due to the local independence structure of the genotypes, recursive factorisation can be used to write the joint genotypic distribution as:

$$
p(\mathbf{W} \mid f)=\prod_{\mathrm{i} \in \mathrm{F}} p\left(\mathbf{w}_{\mathrm{i}} \mid f\right) \prod_{\mathrm{i} \in \mathrm{NF}} p\left(\mathbf{w}_{\mathrm{i}} \mid \mathbf{w}_{\operatorname{sire}(\mathrm{i})}, \mathbf{w}_{\operatorname{dam}(\mathrm{i})}\right)
$$


where $\mathbf{W}=\left(\mathbf{w}_{1}, \ldots, \mathbf{w}_{\mathbf{n}}\right), F$ is the set of founders, and $N F$ is the set of nonfounders.

To fully specify the Bayesian model, improper uniform priors were used for the fixed and genotypic effects [i.e. $p(\mathbf{b}) \propto$ constant, $p(\mathbf{m}) \propto$ constant]. Variance components (i.e. $\sigma_{\mathrm{e}}^{2}$ and $\sigma_{\mathrm{u}}^{2}$ ) were assumed a priori to be independent and to follow the conjugate inverted gamma distribution (i.e. $1 / \sigma_{i}^{2}$ has the prior distribution of a gamma random variable with parameters $\alpha_{\mathrm{i}}$ and $\beta_{\mathrm{i}}$ ). The parameters $\alpha_{\mathrm{i}}$ and $\beta_{\mathrm{i}}$ can be chosen so that the prior distribution has any desired mean and variance. The conjugate Beta prior was used for allele frequency $\left(p(f) \sim \operatorname{Beta}\left(\alpha_{\mathrm{f}}, \beta_{\mathrm{f}}\right)\right)$.

The joint posterior density of all model parameters is proportional to the product of the prior distributions and the conditional distribution of the data, given the parameters:

$$
\begin{aligned}
& P\left(\mathbf{b}, \mathbf{u}, \mathbf{W}, \mathbf{m}, f, \sigma_{\mathrm{e}}^{2}, \sigma_{\mathrm{u}}^{2}, \mathbf{A} \mid \mathbf{y}\right) \propto \\
& p\left(\mathbf{y} \mid \mathbf{b}, \mathbf{u}, \mathbf{W}, \mathbf{m}, \sigma_{\mathrm{e}}^{2}\right) p\left(\sigma_{\mathrm{e}}^{2}\right) p\left(\sigma_{\mathrm{u}}^{2}\right) p\left(\mathbf{u} \mid \sigma_{\mathrm{u}}^{2}\right) p(f) p(\mathbf{W} \mid f) \propto \\
& \exp \left\{-\left[(\mathbf{y}-\mathbf{X} \mathbf{b}-\mathbf{Z} \mathbf{u}-\mathbf{Z W} \mathbf{W})^{\prime}(\mathbf{y}-\mathbf{X} \mathbf{b}-\mathbf{Z u}-\mathbf{Z W} \mathbf{W})+\frac{2}{\beta_{\mathrm{e}}}\right] / 2 \sigma_{\mathrm{e}}^{2}\right\} \\
& \times\left(\sigma_{\mathrm{e}}^{2}\right)^{\left(\frac{n+v_{\mathrm{e}}}{2}+1\right)} \times\left(\sigma_{\mathrm{u}}^{2}\right)^{\left(\frac{q+v_{\mathrm{u}}}{2}+1\right)} \times \exp \left\{-\left[\left(\mathbf{u}^{\prime} \mathbf{A}^{-1} \mathbf{u}\right)+\frac{2}{\beta_{\mathrm{u}}}\right] / 2 \sigma_{\mathrm{u}}^{2}\right\} \\
& \times f^{\alpha_{\mathrm{f}}-1}(1-f)^{\beta_{\mathrm{f}}-1} \times \prod_{i \in F} p\left(\mathbf{w}_{\mathrm{i}} \mid f\right) \times \prod_{i \in N F} p\left(\mathbf{w}_{\mathrm{i}} \mid \mathbf{w}_{\text {sire(i) }}, \mathbf{w}_{\text {dam (i) }}\right)
\end{aligned}
$$

\subsection{Gibbs sampling}

For Bayesian inference, the marginal posterior distribution for the parameters of the model is of interest. With MIMs this requires high dimensional integration and summation of the joint posterior distribution (1), with cannot be expressed in closed form. To perform the integration numerically using the Gibbs sampler requires the construction of a Markov chain which has (1) (normalised) as its stationary distribution. This can be accomplished by defining the transition probabilities of the Markov chain as the full conditional distributions of each model parameter. Samples are then taken from these distributions in an iterative scheme. Each time a full conditional distribution is visited, it is used to sample the corresponding variable, and the realised value is substituted into the conditional distribution of all other variables (see, e.g. [5]).

Instead of updating all variables univariately it is also possible to sample several variables from their joint conditional posterior distribution. Variables that are sampled jointly will be referred to as a 'block'. As long as all variables are sampled, the new Markov chain will still have equation (1) as its stationary distribution.

\subsubsection{Full conditional posterior distributions}

Full conditional distributions were derived from the joint posterior distribution (1). The resulting distributions are presented later. These distributions were also presented by Janss et al. [9], using a slightly different notation. 


\subsubsection{Location parameters}

Hereafter, the restricted additive major gene model will be assumed, such that $\mathbf{m}^{\prime}=(-a, 0, a)$ or $\mathbf{m}=\mathbf{l} a$, where $\mathbf{l}^{\prime}=(-1,0,1)$ and $a$ is the additive effect of the major locus gene. Allowing for genotypic means to vary independently or including a dominance effect entails no difficulty.

The gene effect $(a)$ is considered a classification effect when conditioning on major genotypes $(\mathbf{W})$ and the genetic model at the locus. Consequently, the location parameters in the model are $\boldsymbol{\theta}^{\prime}=\left[\mathbf{b}^{\prime}, a, \mathbf{u}^{\prime}\right]$. Let, $\mathbf{H}=[\mathbf{X}: \mathbf{Z W 1 : Z}]$, $\Omega=\left[\begin{array}{cc}0 & 0 \\ 0 & \mathbf{A}^{-1} k\end{array}\right], k=\sigma_{\mathrm{e}}^{2} / \sigma_{\mathrm{u}}^{2}, C=\left[\mathbf{H}^{\prime} \mathbf{H}+\Omega\right]$, and $\mathbf{m}=\mathbf{l} a$. The posterior distribution of location effects $(\theta)$, given the variance components, major genotypes $(\mathbf{W})$ and data $(\mathbf{y})$ is (following [17]):

$$
\boldsymbol{\theta} \mid \mathbf{W}, \sigma_{\mathrm{u}}^{2}, \sigma_{\mathrm{e}}^{2}, \mathbf{y} \sim \mathrm{N}\left(\widetilde{\boldsymbol{\theta}}, \mathbf{C}^{-1} \sigma_{\mathrm{e}}^{2}\right)
$$

Then, using standard results from multivariate normal theory (e.g. [18] or $[22])$, the full conditional distributions of the parameters in $\theta$ can be written as:

where

$$
\theta_{\mathrm{i}} \mid \theta_{-\mathrm{i}}, \mathbf{W}, \sigma_{\mathrm{u}}^{2}, \sigma_{\mathrm{e}}^{2}, \mathbf{y} \sim \mathrm{n}\left(\widetilde{\boldsymbol{\theta}}_{\mathrm{i}}, \mathbf{C}_{\mathrm{ii}}^{-1} \sigma_{\mathrm{e}}^{2}\right)
$$

$\mathbf{C}_{\mathrm{ii}}$ is the ith diagonal element of $\mathbf{C}, \mathbf{C}_{-\mathrm{i}}$ is the ith row of $\mathbf{C}$ excluding $\mathbf{C}_{\mathrm{ii}}$, and $\mathbf{H}_{\mathbf{i}}$ is the ith column of $\mathbf{H}$.

\subsubsection{Major genotypes}

The full conditional distribution of a given genotype, $\mathbf{w}_{i}$, is found by extracting from equation (1) the terms in which $\mathbf{w}_{\mathrm{i}}$ is present. The probabilities are here given up to a constant of proportionality and must be normalised to ensure that $\sum_{j=1}^{3} p\left(\mathbf{w}_{\mathrm{i}}=\omega_{\mathrm{j}}\right)=1$. The full conditional distribution of genotype $\mathbf{w}_{\mathrm{i}}$ is:

$$
\begin{aligned}
& p\left(\mathbf{w}_{\mathrm{i}}=\omega_{\mathrm{j}} \mid \mathbf{W}_{-\mathrm{i}}, \boldsymbol{\theta}, f, \mathbf{y}\right) \propto \\
& \left(p \left(\mathbf{w}_{\mathrm{i}}=\omega_{\mathrm{j}} \mid f\left(\mathbf{I}_{\mathrm{i} \in \mathrm{F}}+p\left(\mathbf{w}_{\mathrm{i}}=\omega_{\mathrm{j}} \mid \mathbf{w}_{\text {Sire }(\mathrm{i})}, \mathbf{w}_{\text {dam }(\mathrm{i})}\right) \mathbf{I}_{\mathrm{i} \in \mathrm{NF}}\right)\right.\right. \\
& \times p\left(\widetilde{\mathbf{y}}_{\mathrm{i}} \mid \mathbf{w}_{\mathrm{i}}=\omega_{\mathrm{j}}\right) \times \prod_{\mathrm{i}(\mathrm{k}) \in \operatorname{Off}(\mathrm{i})} p\left(\mathbf{w}_{\mathrm{i}(\mathrm{k})} \mid \mathbf{w}_{\mathrm{i}}=\omega_{\mathrm{j}}, \mathbf{w}_{\operatorname{mate}(\mathrm{i}(\mathrm{k}))}\right)
\end{aligned}
$$

where $\mathbf{I}_{\mathrm{i} \in \mathrm{F}}$ and $\mathbf{I}_{\mathrm{i} \in \mathrm{NF}}$ are indicator functions, which are 1 if individual $\mathrm{i}$ is contained in the set of founders (F) or non-founders (NF), respectively, and 0 otherwise. Off $_{(i)}$ is the set of offspring of individual $i$, such that $i(k)$ is the kth offspring of $\mathrm{i}$ resulting from a mating with mate $(\mathrm{i}(\mathrm{k}))$. The terms, $p\left(\mathbf{w}_{\mathrm{i}}=\omega_{\mathrm{j}} \mid p_{1}\right) \mathbf{I}_{\mathrm{I} \in \mathrm{F}}+p\left(\mathbf{w}_{\mathrm{i}}=\omega_{\mathrm{j}} \mid \mathbf{w}_{\text {sire(i) }}, \mathbf{w}_{\text {dam(i) }}\right) \mathbf{I}_{\mathrm{i} \in \mathrm{NF}}$ represent the probability 
of individual i receiving alleles corresponding to genotypes $\omega_{1}, \omega_{2}$ or $\omega_{3}$, and the product over offspring represents the probability of individual i transmitting alleles in the genotypes of the offspring, which are conditioned upon. If individual $\mathrm{i}$ has a phenotypic record, the adjusted record $\widetilde{y}_{\mathrm{i}}=y_{\mathrm{i}}-\mathbf{X}_{\mathrm{i}} \mathbf{b}-\mathbf{Z}_{\mathrm{i}} \mathbf{u}$ contributes the penetrance function:

$$
p\left(\widetilde{y}_{\mathrm{i}} \mid \mathbf{w}_{\mathrm{i}}=\omega_{\mathrm{j}}\right) \propto \exp \left(-\left(\widetilde{y}_{\mathrm{i}}-\omega_{\mathrm{j}} \mathbf{m}\right) / 2 \sigma_{\mathrm{e}}^{2}\right)
$$

where $\mathbf{X}_{\mathbf{i}}$ and $\mathbf{Z}_{\mathrm{i}}$ are the ith rows of the matrices $\mathbf{X}$ and $\mathbf{Z}$.

\subsubsection{Allele frequency}

Conditioning on the sampled genotypes of founder individuals results in contributions of $f$ for each $A_{1}$ sampled and $(1-f)$ for each $A_{2}$ sampled. This is because the sampled genotypes are realisations of the $2 n$ independent Bernoulli $(f)$ random variables used as priors for base population alleles. Multiplying these contributions by the prior $\operatorname{Beta}\left(\alpha_{\mathrm{f}}, \beta_{\mathrm{f}}\right)$ gives

$$
p(f) \propto f^{\left(\alpha_{\mathrm{f}}+n_{A_{1} 1}-1\right)} \times(1-f)^{\left(\beta_{\mathrm{f}}+n_{\left.A_{2}-1\right)}\right.}
$$

where $n_{A_{1}}$ and $n_{A_{2}}$ are the numbers of $A_{1}$ and $A_{2}$ alleles in the base population. The specified distribution is proportional to a $\operatorname{Beta}\left(\alpha_{\mathrm{f}}+n_{A_{1}}, \beta_{\mathrm{f}}+n_{A_{2}}\right)$ distribution. Taking $\alpha_{\mathrm{f}}=\beta_{\mathrm{f}}=1$, the prior on this parameter is a proper uniform distribution.

\subsubsection{Variance components}

The full conditional distribution of the variance component $\sigma_{u}^{2}$ is

$$
p\left(\sigma_{\mathrm{u}}^{2} \mid \mathbf{u}, \mathbf{y}\right) \propto\left(\sigma_{\mathrm{u}}^{2}\right)^{\left(\frac{q+2 \alpha_{\mathrm{u}}}{2}+1\right)} \times \exp \left\{-\left[\left(\mathbf{u}^{\prime} \mathbf{A}^{-1} \mathbf{u}\right)+\frac{2}{\beta_{\mathrm{u}}}\right] / 2 \sigma_{\mathrm{u}}^{2}\right\}
$$

which is proportional to the inverted gamma distribution:

$$
I G\left(\frac{q}{2}+\alpha_{\mathbf{u}}, \frac{1}{\frac{1}{2}\left(\mathbf{u}^{\prime} \mathbf{A}^{-1} \mathbf{u}\right)+\frac{1}{\beta_{\mathbf{u}}}}\right)
$$

Similarly, the full conditional distribution of the variance component $\sigma_{\mathrm{e}}^{2}$ is

$$
\begin{aligned}
& \mathbf{p}\left(\sigma_{\mathrm{e}}^{2} \mid \mathbf{u}, \mathbf{y}\right) \propto\left(\sigma_{\mathrm{e}}^{2}\right)^{\left(\frac{q+2 \alpha_{\mathrm{u}}}{2}+1\right)} \\
& \times \exp \left\{-\left[(\mathbf{y}-\mathbf{X} \mathbf{b}-\mathbf{Z u}-\mathbf{Z W} \mathbf{m})^{\prime}(\mathbf{y}-\mathbf{X} \mathbf{b}-\mathbf{Z} \mathbf{u}-\mathbf{Z W} \mathbf{m})+\frac{2}{\beta_{\mathrm{e}}}\right] / 2 \sigma_{\mathrm{e}}^{2}\right\}
\end{aligned}
$$

which is proportional to the inverted gamma distribution:

$$
I G\left(\frac{n}{2}+\alpha_{\mathrm{e}}, \frac{1}{\frac{1}{2}(\mathbf{y}-\mathbf{X b}-\mathbf{Z} \mathbf{u}-\mathbf{Z W} \mathbf{m})^{\prime}(\mathbf{y}-\mathbf{X} \mathbf{b}-\mathbf{Z u}-\mathbf{Z W m})+\frac{1}{\beta_{\mathrm{e}}}}\right)
$$


The algorithm based on univariate updating can be summarised as follows: I. initiate $\boldsymbol{\theta}, \mathbf{W}, f, \sigma_{\mathrm{u}}^{2}, \sigma_{\mathrm{e}}^{2}$, with legal starting values;

II. sample major genotypes $\mathbf{w}_{\mathrm{i}}$ from equation (3) for $i=\{1, \ldots, q\}$;

III. sample allele frequency from equation (4);

IV. sample location parameters $\theta_{\mathrm{i}}$ (classification effects and polygenic effects) univariately from equation (2), for $i=\left\{1\right.$, dimension $\left._{\theta}\right\}$;

V. sample $\sigma_{u}^{2}$ from equation (5);

VI. sample $\sigma_{\mathrm{e}}^{2}$ from equation (6);

VII. repeat II-VI.

Steps II-VI constitute one iteration. The system is initially monitored until sufficient evidence for convergence is observed. Subsequently, iterations are continued, and the sampled values saved, until the desired precision of features of the posterior distribution has been achieved. The mixing diagnostic used is described in a later section.

\subsection{Blocking strategies}

A more efficient alternative to the univariate updating of variables is to update a set of variables multivariately. Variables updated jointly will be referred to as a 'block'. In this implementation, variables must be sampled from the full conditional distribution of the block. In the present model blocking major genotypes of several individuals alleviates the problems of poor convergence and mixing properties caused by the covariance structure between these variables.

Janss et al. [9] constructed a block for each sire, containing genotypes of the sire and its final offspring. All other individuals were sampled from their full conditional distributions. Janss and co-workers showed that exact calculations needed for these blocks are simple, and this is the first approach we apply in the analysis of the simulated data. However, this blocking strategy only improves the algorithm in pedigree structures with several final offspring. In many applications only a few final offspring exist (e.g. dairy cattle pedigrees), and the blocking calculations become more complicated. Therefore, the second approach applied to the simulated data was to extend the bocking Gibbs sampling algorithm of Jensen et al. [13], using a graphical model representation of genotypes. Here, the conditional distributions of all parameters, other than the major genotypes, are the same regardless of whether blocking is used or not.

\subsubsection{Sire blocking}

In the sire blocking approach, a block is constructed for each sire having final offspring. The blocks contain genotypes of the sire and its final offspring. This requires an exact calculation of the joint conditional genotypic distribution, $p\left(\mathbf{w}_{\mathbf{i}}, \mathbf{w}_{\mathrm{i}(1)}, \ldots, \mathbf{w}_{\mathrm{i}(\mathrm{n}(\mathrm{i}))} \mid \mathbf{W}_{-(\mathrm{i}, \mathrm{i}(\mathrm{l}))}, \boldsymbol{\theta}, \mathbf{y}\right)$, where $\mathrm{i}$ is the index of a sire, $n_{\mathrm{i}}$ denotes the number of final offspring of sire $i$, and the final offspring are indexed by $\mathrm{i}_{(1)}, \mathrm{i}_{(2)}, \ldots, \mathrm{i}\left(\mathrm{n}_{\mathrm{i}}\right)$ or simply $\mathrm{i}(\mathrm{l})$. By definition, this distribution is proportional to $p\left(\mathbf{w}_{\mathrm{i}} \mid \mathbf{W}_{-(\mathrm{i}, \mathrm{i}(\mathrm{l}))}, \boldsymbol{\theta}, \mathbf{y}\right) \times p\left(\mathbf{w}_{\mathrm{i}(1)},\left|, \mathbf{w}_{\mathrm{i}(\mathrm{n}(\mathrm{i}))}\right| \mathbf{w}_{\mathrm{i}}, \mathbf{W}_{-(i, i(l))}, \boldsymbol{\theta}, \mathbf{y}\right)$. Here, the first term is the genotypic distribution of the sire, marginalised with respect to the 
genotypes of the final offspring. In calculating the distribution of the sire's genotype, the three possible genotypes of each offspring are summed over, after weighting each genotype by its relative probability. In this expression, we condition on the mates and the final offspring do not have offspring themselves. Therefore, neighbourhood individuals that contribute to the genotype distribution of the sire are still the same as those in the full conditional distribution. Consequently, the amount of exact calculation needed is linear in the size of the block. The second term is the joint distribution of final offspring genotypes conditional on the sire's genotype. This is equivalent to a product of full conditional distributions of final offspring genotypes because these are conditionally independent, given genotypes of parents.

Even though the final offspring with a common sire are sampled jointly with this sire, the previous discussion shows that this is equivalent to sampling final offspring from their full conditional distributions. Dams and sires with no final offspring are also sampled from their full conditional distributions. This leads to the algorithm proposed by Janss and colleagues which will be referred to as 'sire blocking'.

Sires are sampled according to probabilities:

$$
\begin{aligned}
& p\left(\mathbf{w}_{\mathrm{i}}=\omega_{\mathrm{j}} \mid \mathbf{W}_{-\mathrm{i}, \mathrm{i}(\mathrm{l})}, \boldsymbol{\theta}, f, \mathbf{y}\right) \propto\left(p\left(\mathbf{w}_{\mathrm{i}}=\omega_{\mathrm{i}} \mid f\right) \mathbf{I}_{\mathrm{F}}+p\left(\mathbf{w}_{\mathrm{i}}=\omega_{\mathrm{j}} \mid \mathbf{w}_{\text {sire }(\mathrm{i})}, \mathbf{w}_{\text {dam }(\mathrm{i})}\right) \mathbf{I}_{\mathrm{NF}}\right) \\
& \times p\left(\widetilde{\mathbf{y}}_{\mathrm{i}} \mathbf{w}_{\mathrm{i}}=\boldsymbol{\omega}_{\mathrm{j}}\right) \times \prod_{\mathrm{i}(\mathrm{k}) \in \operatorname{NonFinal}(\mathrm{i})} p\left(\mathbf{w}_{\mathrm{i}(\mathrm{k})} \mid \mathbf{w}_{\mathrm{i}}=\omega_{\mathrm{j}}, \mathbf{w}_{\operatorname{dam}(\mathrm{i}(\mathrm{k}))}\right) \\
& \times \prod_{\mathrm{i}(\mathrm{k}) \in \text { Final }(\mathrm{i})} \sum_{\mathrm{m}=\mathrm{l}}^{3} p\left(\mathbf{w}_{\mathrm{i}(\mathrm{l})}=\boldsymbol{\omega}_{\mathrm{m}} \mid \mathbf{w}_{\mathrm{i}}=\boldsymbol{\omega}_{\mathrm{j}}, \mathbf{w}_{\text {dam }(\mathrm{i}(\mathrm{l}))}\right) \times p\left(\widetilde{\mathbf{y}}_{\mathrm{i}(\mathrm{l})} \mid \mathbf{w}_{\mathrm{i}(\mathrm{l})}=\omega_{\mathrm{m}}\right)
\end{aligned}
$$

where Final(i) is the set of final offspring of sire i, and NonFinal(i) is the set of non-final offspring.

Dams are sampled according to equation (3), and final offspring according to:

$$
p\left(\mathbf{w}_{\mathrm{i}(1)}=\omega_{\mathrm{m}}\right) \propto p\left(\mathbf{w}_{\mathrm{i}(1)} \mid \mathbf{w}_{\mathrm{i}}, \mathbf{w}_{\mathrm{dam}(\mathrm{i}(1))}\right) \times p\left(\widetilde{y}_{\mathrm{i}(1)} \mid \mathbf{w}_{\mathrm{i}(1)}=\omega_{\mathrm{m}}\right)
$$

Again, the probabilities must be normalised. The sire blocking strategy is then constructed as in the previous algorithm, except that step II is replaced by the following: if individual $i$ is a sire, sample genotype from equation (7), followed by sampling of final offspring $i(l)$ from equation (8). If individual $i$ is a dam, sample genotype from equation (3).

\subsubsection{General blocking using graph theory}

This approach involves a more general blocking strategy by representing major genotypes in a graphical model. This representation enables the formation of optimal blocks, each containing the majority of genotypes. The blocks are formed so that exact calculations in each block are possible. These exact calculations can be used to obtain a random sample from the full conditional distribution of the block.

In general, the methods described later can be used to perform exact calculations in a posterior distribution, denoted here by $p(\mathbf{V} \mid \mathbf{e})$, where $\mathbf{V}$ 
denotes the variables of the Bayesian network, and $\mathbf{e}$ is called 'evidence'. The evidence can contain both the data $(\mathbf{y})$, on which $\mathbf{V}$ has a causal effect, and other known parameters. In turn, the posterior distribution is written as the joint prior of $\mathrm{V}$ multiplied by the conditional distribution of evidence $[p(\mathbf{V} \mid \mathbf{e}) \propto p(\mathbf{V}) p(\mathbf{e} \mid \mathbf{V})]$

Jensen et al. [13] used the Bayesian network representation as the basis of their blocking Gibbs sampling algorithm for a single locus model. In their model, $\mathbf{V}$ contained the discrete genotypes and $\mathbf{e}$ the data, which were assumed to be completely determined by the genotypes. However, MIMs are more complex, as they contain several variables in addition to the major genotypes (e.g. systematic and random environmental effects as well as correlated polygenic effects affect phenotypes). Consequently, the representation of Jensen et al. [13] cannot be used directly for MIMs.

To incorporate the extra parameters of the model, a Gibbs sampling algorithm is constructed in which the continuous variables pertaining to the MIM are sampled from their full conditional densities. In each round the sampled realisations can then be inserted as evidence in the Bayesian network. This algorithm requires the Bayesian network representation of major genotypes $(\mathbf{V} \equiv \mathbf{W})$, with data and continuous variables as evidence $\left(\mathbf{e}=\mathbf{b}, \mathbf{u}, \mathbf{m}, f, \sigma_{\mathrm{e}}^{2}\right.$, $\left.\sigma_{u}^{2}, \mathbf{y}\right)$. However, because an exact calculation of the joint distribution of all genotypes is not possible, a small number of blocks (e.g. $\left.\mathbf{B}_{1}, \mathbf{B}_{2}, \ldots, \mathbf{B}_{5}\right)$ are constructed, and for each block a Bayesian network $\mathrm{BN}_{\mathrm{i}}$ is defined. For each $\mathrm{BN}_{\mathrm{i}}$, let the variables be the genotypes in the block $\mathbf{V} \equiv \mathbf{B}_{\mathrm{i}}$. Further, let the evidence be genotypes in the complementary set $\left(\mathbf{B}_{i}^{\mathrm{c}}=\mathbf{W} \backslash \mathbf{B}_{\mathbf{i}}\right)$, realised values of other variables, and the data [i.e. $\left.\mathbf{e}=\left(\mathbf{B}_{\mathrm{i}}^{\mathbf{c}}, \mathbf{b}, \mathbf{u}, \mathbf{m}, \boldsymbol{\sigma}_{\mathrm{e}}^{2}, \boldsymbol{\sigma}_{\mathrm{u}}^{2}, f, \mathbf{y}\right)\right]$. These Bayesian networks are a graphical representation of the joint conditional distribution of all major genotypes within a block, given the complementary set, all other continuous variables, and the data $\left(p\left(\mathbf{B}_{\mathrm{i}} \mid \mathbf{B}_{\mathrm{i}}^{\mathrm{c}}, \mathbf{b}, \mathbf{u}, \mathbf{m}, f, \boldsymbol{\sigma}_{\mathrm{e}}^{2}, \sigma_{\mathrm{u}}^{2}, \mathbf{y}\right)\right)$. This is equivalent to a Bayesian network, where data corrected for the current values of all continuous variables are inserted as evidence [i.e. $p\left(\mathbf{B}_{\mathrm{i}} \mid \mathbf{B}_{\mathrm{i}}^{\mathrm{c}}, \mathbf{b}, \mathbf{u}, \mathbf{m}, f, \sigma_{\mathrm{e}}^{2}, \sigma_{\mathrm{u}}^{2}, \mathbf{y}\right) \propto$ $\left.p\left(\mathbf{B}_{\mathrm{i}}\right) * p\left(\mathbf{y} \mid \mathbf{W}, \mathbf{b}, \mathbf{u}, \mathbf{m}, f, \sigma_{\mathrm{e}}^{2}, \sigma_{\mathrm{u}}^{2}\right)=p\left(\mathbf{B}_{\mathrm{i}}\right) * p\left(\tilde{\mathbf{y}} \mid \mathbf{B}_{\mathrm{i}}^{\mathrm{c}}, f\right)\right]$. The last term is described as the penetrance function underneath equation (3).

In the following sections, some details of the graphical model representation are described. This is not intended to be a complete description of graphical models, which is a very comprehensive area of which more details can be found in, e.g. [14-16]. The following is rather meant to focus on operations used in the current work.

\subsubsection{Bayesian networks}

A Bayesian network is a graphical representation of a set of random variables, V, which can be organised, in a directed acyclic graph (e.g. [14]) (figure 1a). A graph is directed when for each pair of neighbouring variables, one variable is causally dependent on the other, but not vice versa. These causal dependencies between variables are represented by directed links which connect them. The graph is acyclic if, following the direction of the directed links, it is not possible to return to the same variable. Variables with causal links pointing to $v_{\mathrm{i}}$ are denoted as parents of $v_{\mathrm{i}}\left[p a\left(v_{\mathrm{i}}\right)\right]$. Should $v_{\mathrm{i}}$ have parents, the conditional probability distribution $p\left(v_{\mathrm{i}} \mid p a\left(v_{\mathrm{i}}\right)\right)$ is associated with it. However, should $v_{\mathrm{i}}$ 


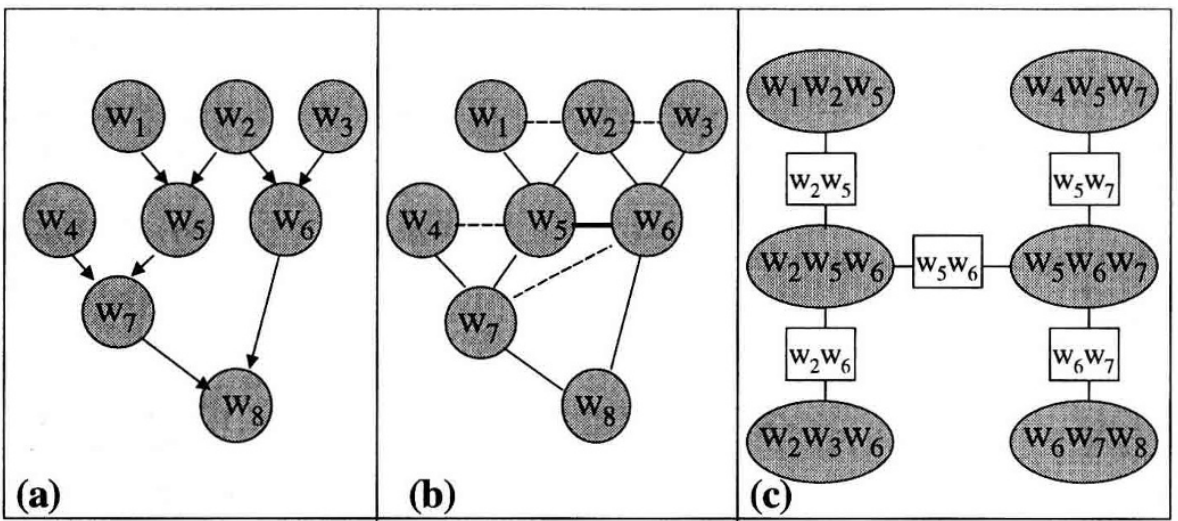

Figure 1. Compilation of a Bayesian network (a) into a junction tree (c) via the triangulated graph (b).

have no parents, this reduces to the unconditional prior distribution $p\left(v_{\mathrm{i}}\right)$. The joint distribution is written $p(V)=\prod_{\mathrm{i}} p\left(v_{\mathrm{i}} \mid p a\left(v_{\mathrm{i}}\right)\right)$.

In this study the variables in the network represent a major genotype, $\mathbf{w}_{\mathrm{i}}$. The links pointing from parents to offspring represent probabilities of alleles being transmitted from parents to offspring. Therefore, the conditional distributions associated with variables are the Mendelian segregation probabilities $\left(p\left(\mathbf{w}_{\mathrm{i}} \mid \mathbf{w}_{\mathrm{s}}, \mathbf{w}_{\mathrm{d}}\right)\right)$. A simple pedigree is depicted in figure $1 a$ as a Bayesian network. From this, it is apparent that a pedigree of genotypes is a special case of a Bayesian network.

In general, exact computations among the genotypes are required. For example, in figure $1 a$ should it be required to calculate $p\left(\mathbf{w}_{1}, \mathbf{w}_{2}, \mathbf{w}_{5}\right)$, this can be carried out as: $p\left(\mathbf{w}_{1}, \mathbf{w}_{2}, \mathbf{w}_{5}\right)=\sum_{\mathbf{w}_{3}, \mathbf{w}_{4}, \mathbf{w}_{6}, \mathbf{w}_{7}, \mathbf{w}_{8}} p\left(\mathbf{w}_{1}, \mathbf{w}_{2}, \mathbf{w}_{3} \mathbf{w}_{4}, \mathbf{w}_{5}, \mathbf{w}_{6} \mathbf{w}_{7}, \mathbf{w}_{8}\right)$.

The size of the probability table increases exponentially with the number of genotypes. Therefore, it rapidly increases to sizes that are not manageable. However, by using the local independence structure, recursive factorisation allows us to write the desired distribution as:

$$
\begin{aligned}
p\left(\mathbf{w}_{1}, \mathbf{w}_{2}, \mathbf{w}_{5}\right)= & \sum_{\mathbf{w}_{3}, \mathbf{w}_{4}, \mathbf{w}_{6}, \mathbf{w}_{7}, \mathbf{w}_{8}} p\left(\mathbf{w}_{1}\right) p\left(\mathbf{w}_{2}\right) p\left(\mathbf{w}_{5} \mid \mathbf{w}_{1}, \mathbf{w}_{2}\right) p\left(\mathbf{w}_{3}\right) p\left(\mathbf{w}_{6} \mid \mathbf{w}_{2}, \mathbf{w}_{3}\right) p\left(\mathbf{w}_{4}\right) \\
& p\left(\mathbf{w}_{7} \mid \mathbf{w}_{4}, \mathbf{w}_{5}\right) p\left(\mathbf{w}_{8} \mid \mathbf{w}_{6}, \mathbf{w}_{7}\right) \\
= & p\left(\mathbf{w}_{1}\right) p\left(\mathbf{w}_{2}\right) p\left(\mathbf{w}_{5} \mid \mathbf{w}_{1}, \mathbf{w}_{2}\right) \\
& \sum_{\mathbf{w}_{3}, \mathbf{w}_{6}} p\left(\mathbf{w}_{3}\right) p\left(\mathbf{w}_{6} \mid \mathbf{w}_{2}, \mathbf{w}_{3}\right) \sum_{\mathbf{w}_{4}, \mathbf{w}_{7}} p\left(\mathbf{w}_{4}\right) p\left(\mathbf{w}_{7} \mid \mathbf{w}_{4}\right) \sum_{\mathbf{w}_{8}} p\left(\mathbf{w}_{8} \mid \mathbf{w}_{6}, \mathbf{w}_{7}\right)
\end{aligned}
$$

This is much more efficient in terms of storage requirements and describes the general idea underlying methods for exact computations of posterior distributions in Bayesian networks. When the Bayesian network contains loops, it is difficult to set the order of summations such that the sizes of the probability 
tables are minimised. Therefore, an algorithm is required. The method of 'peeling' by Elston and Stewart [4], and generalised by Cannings et al. [2], provides algorithms for performing such calculations with genetic applications. However, for other operations needed in the blocked Gibbs sampling algorithm, peeling cannot be used. Instead, we use the algorithm of Lauritzen and Spiegelhalter [16], which also is based on the above ideas. This algorithm transforms the Bayesian network into a so-called junction tree.

\subsubsection{The junction tree}

The junction tree is a secondary structure of the Bayesian network. This structure generates a posterior distribution that is mathematically identical to the posterior distribution in the Bayesian network. However, properties of the junction tree greatly reduce the required computations. The desired properties are fulfilled by any structure that satisfies the following definition.

Definition 1 (junction tree). A junction tree is a graph of clusters. The clusters, also called cliques, $\left(C_{\mathrm{i}}, \mathrm{i}=1, n_{\mathrm{i}}\right)$ are subsets of $\mathbf{V}$, and the union of all cliques is $\mathbf{V}:\left(C_{1} \cup C_{2} \cup, \ldots, \cup C_{\mathbf{i}}=\mathbf{V}\right)$. The cliques are organised into a graph with no loops (cycles), and by following the path between neighbouring cliques it is not possible to return to the same clique. Between each pair of neighbouring cliques is a separator, $S$, which contains the intersection of the two cliques $\left(S_{12}=C_{1} \cup C_{2}\right)$. Finally, the intersection of any two cliques, $C_{\mathrm{i}}$ and $C_{\mathrm{j}}$, is present in all cliques and separators on the unique path between $C_{\mathrm{i}}$ and $C_{\mathrm{j}}$.

\subsubsection{Transformation of a Bayesian network into a junction tree}

In general, there is no unique junction tree for a given Bayesian network. However, the algorithm of Lauritzen and Spiegelhalter [16] generates a junction tree for any Bayesian network with the property that the cliques generally become as small as possible. This is important as small cliques make calculations more efficient. In the following section, we introduce some basic operations of that algorithm, transforming the Bayesian network shown in figure $1 a$ into a junction tree.

The network is first turned into an undirected graph, by removing the directions of the links. Links are then added between parents. The added links (seen in figure $1 b$ as the dashed links) are denoted 'moral links', and the resulting graph is called the 'moral graph'. The next step is to 'triangulate' the graph. If cycles of length greater than three exist, and no other links connect variables in that cycle, extra 'fill-in links' must be added until no such cycles exist. After links are added between parents, as shown in figure 1, there is a cycle of length four which contains the variables $\mathbf{w}_{2}, \mathbf{w}_{5}, \mathbf{w}_{7}$ and $\mathbf{w}_{6}$. An extra fill-in link must be added either between $\mathbf{w}_{2}$ and $\mathbf{w}_{7}$ or as shown with the thick link between $\mathbf{w}_{5}$ and $\mathbf{w}_{6}$. Finally, from the triangulated graph, the junction tree is established by identifying all 'cliques'. These are defined as maximal sets of variables that are all pairwise linked. In other words, a set of variables that are all pairwise connected by links must be in the same clique. These cliques must be arranged into a graph with no loops, in such a way, that for each pair of cliques $C_{\mathrm{i}}, C_{\mathrm{j}}$, all cliques and separators on the unique path between $C_{\mathrm{i}}$ and 
$C_{\mathrm{j}}$ contain the intersection $C_{\mathrm{i}} \cap C_{\mathrm{j}}$. This requirement ensures that variables in $C_{\mathrm{i}}$ and $C_{\mathrm{j}}$ are conditionally independent, given variables on the path between them.

\subsubsection{Exact computations in a junction tree}

To perform exact calculations, the junction tree is initialised by constructing belief tables for all cliques $\left(B\left(C_{1}\right), \ldots, B\left(C_{\mathrm{n}(\mathrm{c})}\right)\right)$ and separators $\left(B\left(S_{1}\right), \ldots, B\left(S_{\mathrm{n}(\mathrm{s})}\right)\right)$. Each belief table conforms to the joint probability distribution of variables in that clique or separator, and contains the current belief of the joint posterior distribution of these variables. This is also called the belief potential of these cliques/separators. For example, $B\left(C_{1}\right)$ represents $p\left(C_{1} \mid \mathbf{y}\right)$ in figure 2a. In the following we assume that individual 8 in figure 1a has a phenotypic record. Then, the belief tables are initialised by first setting all entries in each belief table to one. Prior probabilities of variables without parents are then multiplied onto exactly one arbitrarily chosen clique in which the variable is present. Finally, the conditional probabilities of variables with parents are multiplied onto the unique clique which contains that variable and its parents. Following this procedure, the junction tree in figure $1 \mathrm{c}$ could be initialised as follows: $C_{1}=\left(\mathbf{w}_{1}, \mathbf{w}_{2}, \mathbf{w}_{5}\right)$ is initialised with $B\left(C_{1}\right)=$ $p\left(\mathbf{w}_{1}\right) p\left(\mathbf{w}_{2}\right) p\left(\mathbf{w}_{5} \mid \mathbf{w}_{1}, \mathbf{w}_{2}\right), C_{2}=\left(\mathbf{w}_{2}, \mathbf{w}_{5}, \mathbf{w}_{6}\right)$ is initialised with all ones for $B\left(C_{2}\right), C_{3}=\left(\mathbf{w}_{2}, \mathbf{w}_{3}, \mathbf{w}_{6}\right)$ is initialised with $B\left(C_{3}\right)=p\left(\mathbf{w}_{3}\right) p\left(\mathbf{w}_{6} \mid \mathbf{w}_{2}, \mathbf{w}_{3}\right)$, $C_{4}=\left(\mathbf{w}_{4}, \mathbf{w}_{5}, \mathbf{w}_{7}\right)$ is initialised with $B\left(C_{4}\right)=p\left(\mathbf{w}_{4}\right) p\left(\mathbf{w}_{7} \mid \mathbf{w}_{4}, \mathbf{w}_{5}\right), C_{5}=$ $\left(\mathbf{w}_{5}, \mathbf{w}_{6}, \mathbf{w}_{7}\right)$ is initialised with all ones for $B\left(C_{5}\right), C_{6}=\left(\mathbf{w}_{6}, \mathbf{w}_{7}, \mathbf{w}_{8}\right)$ is initialised with $B\left(C_{6}\right)=p\left(\mathbf{w}_{8} \mid \mathbf{w}_{6}, \mathbf{w}_{7}\right) p\left(\mathbf{y}_{8} \mid \mathbf{w}_{8}\right)$, and separators are initialised with all ones. After having initialised the junction tree in this way, we note that the product of the belief potentials for all cliques is equal to the joint posterior distribution of all variables:

$$
\begin{aligned}
& B\left(C_{1}\right) \times B\left(C_{2}\right) \times B\left(C_{3}\right) \times B\left(C_{4}\right) \times B\left(C_{5}\right) \times B\left(C_{6}\right) \\
&= p\left(\mathbf{w}_{1}\right) p\left(\mathbf{w}_{2}\right) p\left(\mathbf{w}_{5} \mid \mathbf{w}_{1}, \mathbf{w}_{2}\right) p\left(\mathbf{w}_{3}\right) p\left(\mathbf{w}_{6} \mid \mathbf{w}_{2}, \mathbf{w}_{3}\right) p\left(\mathbf{w}_{4}\right) \\
& p\left(\mathbf{w}_{7} \mid \mathbf{w}_{4}, \mathbf{w}_{5}\right) p\left(\mathbf{w}_{8} \mid \mathbf{w}_{6}, \mathbf{w}_{7}\right) p\left(\mathbf{y}_{8} \mid \mathbf{w}_{8}\right) \\
&= P\left(\mathbf{w}_{1}, \mathbf{w}_{2}, \mathbf{w}_{3}, \mathbf{w}_{4}, \mathbf{w}_{5}, \mathbf{w}_{6}, \mathbf{w}_{7}, \mathbf{w}_{8} \mid \mathbf{y}_{8}\right)
\end{aligned}
$$

The general rule of this property is given by:

$$
\frac{\prod_{\mathbf{i}} B\left(C_{\mathrm{i}}\right)}{\prod_{\mathbf{j}} B\left(S_{\mathbf{j}}\right)}=p(\mathbf{W} \mid \mathbf{y})
$$

\subsubsection{Junction tree propagation}

The initialisation described in the previous section is arbitrary in the sense that $p\left(\mathbf{w}_{2}\right)$ could have been multiplied onto $B\left(C_{2}\right)$ instead of $B\left(C_{1}\right)$. Therefore, the belief tables do not at this point reflect the knowledge of variables in the corresponding cliques. This is only so after each belief table has been updated 


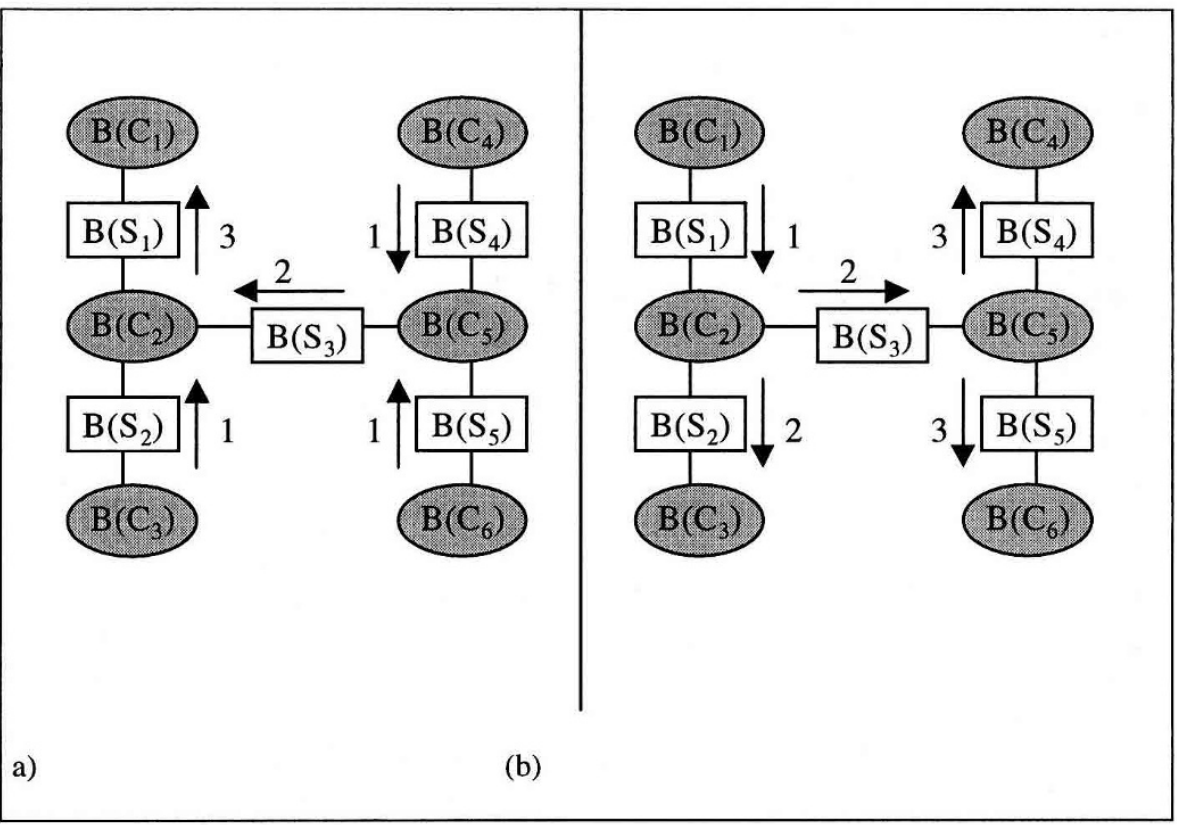

Figure 2. Belief potentials of the cliques, $B(C)$, and separators, $B(S)$, of the junction tree in figure 1c. Arrows indicate direction and order of absorptions in a call of collect evidence (a) and a call of distribute evidence (b) from the clique $C_{1}$.

with the information on all other variables. Propagation in junction trees is a means of updating the belief with such information.

This updating is performed by means of an operation called 'absorption'. This has the effect of propagating information between neighbouring cliques. For example, if information is propagated from $B\left(C_{6}\right)$ to $B\left(C_{5}\right)$ as in figure 2, $B\left(C_{5}\right)$ is said to absorb from $B\left(C_{6}\right)$, or, equivalently, $C_{6}$ is said to send a message to $C_{5}$. The absorption operation consists of the following calculation: $B^{*}\left(C_{5}\right)=B\left(C_{5}\right) \frac{B^{*}\left(S_{5}\right)}{B\left(S_{5}\right)}$, where $B^{*}\left(S_{5}\right)=\sum_{C_{6} \backslash S_{5}} B\left(C_{6}\right)$. The absorption can be regarded as updating the belief potential of $p\left(\mathbf{w}_{5}, \mathbf{w}_{6}, \mathbf{w}_{7}\right)\left(B\left(\mathbf{w}_{5}, \mathbf{w}_{6}, \mathbf{w}_{7}\right)\right)$ with information on the belief potential of $p\left(\mathbf{w}_{6}, \mathbf{w}_{7}, \mathbf{w}_{8}\right)\left(B\left(\mathbf{w}_{6}, \mathbf{w}_{7}, \mathbf{w}_{8}\right)\right)$. This is accomplished by first finding the conditional belief of variables in $C_{5}$ given variables in $C_{6}$ by $B\left(\mathbf{w}_{5} \mid \mathbf{w}_{6}, \mathbf{w}_{7}\right)=\frac{B\left(\mathbf{w}_{5}, \mathbf{w}_{6}, \mathbf{w}_{7}\right)}{B\left(\mathbf{w}_{6}, \mathbf{w}_{7}\right)}$. The joint belief of variables in $C_{5}$ is then updated with new information from $C_{6}$ by $B^{*}\left(\mathbf{w}_{5}, \mathbf{w}_{6}, \mathbf{w}_{7}\right)=$ $B^{*}\left(\mathbf{w}_{6}, \mathbf{w}_{7}\right) B\left(\mathbf{w}_{5} \mid \mathbf{w}_{6}, \mathbf{w}_{7}\right)$, where $B^{*}\left(\mathbf{w}_{6}, \mathbf{w}_{7}, \mathbf{w}_{8}\right)$.

The junction tree is invariant to the absorptions. This means that after an absorption, equation (11) is still true.

The object is now to perform a sequence of absorptions. In this study, sequences are defined by the call of the routines 'collect evidence', and 'distribute evidence' [15]. Collect evidence is an operation that collects all evidence in the 
junction tree towards a single clique. Consequently, calling collect evidence from any clique results in the belief table being equivalent to the joint posterior distribution of the variables it contains. As an example, figure 2 shows that calling collect evidence from $C_{1}$ results in: $B\left(C_{1}\right) \propto p\left(\mathbf{w}_{1}, \mathbf{w}_{2}, \mathbf{w}_{5} \mid \mathbf{y}_{8}\right)$, and the order of absorptions is established as follows. First, $C_{1}$ requests to absorb information from its neighbours $\left(C_{2}\right)$. However, this operation is only allowed if $C_{2}$ has already absorbed from all its other neighbours $\left(C_{3}\right.$ and $\left.C_{5}\right)$. Since this is not the case, $C_{2}$ will recursively request for absorption from these cliques. This is granted for $C_{3}$, but $C_{5}$ still has not absorbed from $C_{4}$ and $C_{6}$, which it requested. This is finally granted, and the absorptions can be performed in the order illustrated in figure $2 a$.

Distribute evidence from $C_{1}$ in figure $2 b$ is performed by allowing $C_{1}$ to send a message to all its neighbours. When a clique has received a message it will send a new message to all of its neighbours, except to the clique it has just received a message from. In our example the order of messages (absorptions) is illustrated in figure $2 b$.

If 'collect evidence' is followed by the routine 'distribute evidence' from the same clique, then for any clique $B\left(C_{\mathrm{i}}\right) \propto p\left(C_{\mathrm{i}} \mid \mathbf{y}\right)[15]$. This is a very attractive property because it is then possible to find the marginal posterior density of any variable, by summing other variables out of any clique in which it is present, rather than summing all other variables out of the joint distribution.

\subsubsection{Example of exact calculations}

An example is provided in this section to illustrate the relationship between exact calculations with or without the use of the junction tree representation.

Should we want to compute the marginal posterior probability distribution $p\left(\mathbf{w}_{1} \mid y_{8}\right)$, this can be carried out directly using standard methods of probability:

$$
p\left(\mathbf{w}_{1} \mid \mathbf{y}_{8}\right) \propto \sum_{\mathbf{w}_{2}, \ldots, \mathbf{w}_{8}} p\left(\mathbf{w}_{1}, \mathbf{w}_{2}, \mathbf{w}_{3}, \mathbf{w}_{4}, \mathbf{w}_{5}, \mathbf{w}_{6}, \mathbf{w}_{7}, \mathbf{w}_{8}\right) p\left(\mathbf{y}_{8} \mid \mathbf{w}_{8}\right)
$$

However, the independence structure between genotypes allows for recursive factorisation:

$$
\begin{array}{r}
p\left(\mathbf{w}_{1}, \mathbf{w}_{2}, \mathbf{w}_{3}, \mathbf{w}_{4}, \mathbf{w}_{5}, \mathbf{w}_{6}, \mathbf{w}_{7}, \mathbf{w}_{8} \mid \mathbf{y}_{8}\right)=p\left(\mathbf{w}_{1}\right) p\left(\mathbf{w}_{2}\right) p\left(\mathbf{w}_{5} \mid \mathbf{w}_{1}, \mathbf{w}_{2}\right) \\
p\left(\mathbf{w}_{3}\right) p\left(\mathbf{w}_{6} \mid \mathbf{w}_{2}, \mathbf{w}_{3}\right) p\left(\mathbf{w}_{4}\right) p\left(\mathbf{w}_{7} \mid \mathbf{w}_{4}, \mathbf{w}_{5}\right) p\left(\mathbf{w}_{8} \mid \mathbf{w}_{6}, \mathbf{w}_{7}\right) p\left(\mathbf{y}_{8} \mid \mathbf{w}_{8}\right)
\end{array}
$$

and we can write equation (9) as:

$$
\begin{aligned}
p\left(\mathbf{w}_{1} \mid \mathbf{y}_{8}\right) \propto \sum_{\mathbf{w}_{2}, \mathbf{w}_{5}}\left\{p ( \mathbf { w } _ { 1 } ) p ( \mathbf { w } _ { 2 } ) p ( \mathbf { w } _ { 5 } | \mathbf { w } _ { 1 } , \mathbf { w } _ { 2 } ) \sum _ { \mathbf { w } _ { 6 } } \left(\sum_{\mathbf{w}_{3}} p\left(\mathbf{w}_{3}\right) p\left(\mathbf{w}_{6}, \mid \mathbf{w}_{2}, \mathbf{w}_{3}\right)\right.\right. \\
\left.\left.\sum_{\mathbf{w}_{7}}\left[\sum_{\mathbf{w}_{4}} p\left(\mathbf{w}_{4}\right) p\left(\mathbf{w}_{7} \mid \mathbf{w}_{4}, \mathbf{w}_{5}\right)\right]\left[\sum_{\mathbf{w}_{8}} p\left(\mathbf{w}_{8} \mid \mathbf{w}_{6}, \mathbf{w}_{7}\right) p\left(\mathbf{y}_{8} \mid \mathbf{w}_{8}\right)\right]\right)\right\}
\end{aligned}
$$

The junction tree algorithm is then used as follows. First, the junction tree in figure $1 \mathrm{c}$ is formed and initialised by the method shown previously. Collect 
evidence is then called from $C_{1}$ to calculate $p\left(C_{1} \mid \mathbf{y}_{8}\right)$. As already described, this call consists of the series of absorptions ordered as illustrated in figure $2 a$. The corresponding calculations are as follows. First, absorptions indicated by 1 in figure 2a: $B^{*}\left(C_{5}\right)=B\left(C_{5}\right) \frac{B^{*}\left(S_{4}\right)}{B\left(S_{4}\right)} \frac{B^{*}\left(S_{5}\right)}{B\left(S_{5}\right)}$, where $B^{*}\left(S_{4}\right)=\sum_{\mathbf{w}_{4}} B\left(C_{4}\right)$ and $B^{*}\left(S_{5}\right)=\sum_{\mathbf{w}_{8}} B\left(C_{8}\right)$ and $B^{*}\left(C_{2}\right)=B\left(C_{2}\right) \frac{B^{*}\left(S_{2}\right)}{B\left(S_{2}\right)}$, where $B^{*}\left(S_{2}\right)=\sum_{\mathbf{w}_{3}} B\left(C_{3}\right)$. Second, absorptions indicated by 2 in figure $2 a: B^{* *}\left(C_{2}\right)=B\left(C_{2}\right) \frac{B^{*}\left(S_{3}\right)}{B\left(S_{3}\right)}$, where $B^{*}\left(S_{3}\right)=\sum_{\mathbf{w}_{7}} B^{*}\left(C_{5}\right)$. Finally, absorptions indicated by 3 in figure $2 a$ : $B^{*}\left(C_{1}\right)=B\left(C_{1}\right) \frac{B^{*}\left(S_{1}\right)}{B\left(S_{1}\right)}$, where $B^{*}\left(S_{1}\right)=\sum_{\mathbf{w}_{6}} B^{*}\left(C_{2}\right)$.

After collect evidence has been completed, $p\left(\mathbf{w}_{1} \mid \mathbf{y}\right)$ can be found by $p\left(\mathbf{w}_{1} \mid \mathbf{y}\right) \propto \sum_{\mathbf{w}_{2} \mathbf{w}_{5}} B^{*}\left(C_{1}\right)$. Writing these calculations together, and substituting the initial probabilities (without the tables of all ones), we obtain:

$$
\begin{aligned}
& p\left(\mathbf{w}_{1} \mid \mathbf{y}\right) \propto \sum_{\mathbf{w}_{2}, \mathbf{w}_{5}} B\left(C_{1}\right) \\
& \left\{\sum_{\mathbf{w}_{6}} B\left(C_{2}\right)\left(\sum_{\mathbf{w}_{3}} B\left(C_{3}\right)\right)\left(\sum_{\mathbf{w}_{7}} B\left(C_{5}\right)\left[\sum_{\mathbf{w}_{4}} B\left(C_{4}\right)\right]\left[\sum_{\mathbf{w}_{8}} B\left(C_{6}\right)\right]\right)\right\} \\
& p\left(\mathbf{w}_{1} \mid \mathbf{y}_{8}\right) \propto \sum_{\mathbf{w}_{2}, \mathbf{w}_{5}}\left\{p ( \mathbf { w } _ { 1 } ) p ( \mathbf { w } _ { 2 } ) p ( \mathbf { w } _ { 5 } | \mathbf { w } _ { 1 } , \mathbf { w } _ { 2 } ) \sum _ { \mathbf { w } _ { 6 } } \left(\sum_{\mathbf{w}_{3}} p\left(\mathbf{w}_{3}\right) p\left(\mathbf{w}_{6} \mid \mathbf{w}_{2}, \mathbf{w}_{3}\right)\right.\right. \\
& \left.\left.\sum_{\mathbf{w}_{7}}\left[\sum_{\mathbf{w}_{4}} p\left(\mathbf{w}_{4}\right) p\left(\mathbf{w}_{7} \mid \mathbf{w}_{4}, \mathbf{w}_{5}\right)\right]\left[\sum_{\mathbf{w}_{8}} p\left(\mathbf{w}_{8} \mid \mathbf{w}_{6}, \mathbf{w}_{7}\right) p\left(\mathbf{y}_{8} \mid \mathbf{w}_{8}\right)\right]\right)\right\}
\end{aligned}
$$

This is exactly the same calculation as equation (10), which illustrates that junction tree propagation is basically a method to separate calculations into smaller steps, and to arrange the order of these, such that the correct result is obtained.

\subsubsection{Random propagation}

Another propagation algorithm, which relies on the junction tree structure, is 'random propagation', developed by Dawid [3]. This method provides a random sample from the joint posterior distribution of all variables in the Bayesian network, $p(\mathbf{V} \mid \mathbf{e})$. Random propagation is initialised by calling collect evidence from an arbitrarily chosen clique $C_{0}$. As mentioned previously, this results in $B\left(C_{0}\right)$ being equal to the joint posterior distribution of variables contained in $C_{0},\left(B\left(C_{0}\right) \propto P\left(C_{0} \mid \mathbf{e}\right)\right) . B\left(C_{0}\right)$ is then used to sample the variables in $C_{0}$. Information on the realised state of variables is distributed to the 
neighbouring cliques $\left(C_{\mathrm{n}}\right)$, by absorption from $C_{0}$ to $C_{\mathrm{n}}$. The belief tables of $C_{\mathrm{n}}$ will then be proportional to the joint posterior distribution of variables contained in the given cliques, conditional on the variables already sampled. That is, $B\left(C_{\mathrm{n}}\right) \propto p\left(C_{\mathrm{n}} \mid C_{0}, \mathbf{e}\right)=p\left(C_{\mathrm{n}} \backslash\left\{C_{0} \cap C_{\mathrm{n}}\right\} \mid C_{0}, \mathbf{e}\right)$. After normalisation, variables of $C_{\mathrm{n}} \backslash\left\{C_{0} \cap C_{\mathrm{n}}\right\}$ are sampled, and absorptions are performed to their neighbouring cliques. Sampling and sending messages is continued in this manner until the entire network is sampled. The order in which sampling is performed follows the order of messages in distribute evidence (figure $2 b$ ). In our genetic example, we can first collect evidence to $C_{1}$. Performing the random propagation algorithm then involves sampling from the following distributions:

$$
\begin{aligned}
& p\left(\mathbf{w}_{1}, \mathbf{w}_{2}, \mathbf{w}_{5} \mid \mathbf{y}\right) \times p\left(\mathbf{w}_{6} \mid \mathbf{w}_{2}, \mathbf{w}_{5}, \mathbf{y}\right) \times p\left(\mathbf{w}_{3} \mid \mathbf{w}_{2}, \mathbf{w}_{6}, \mathbf{y}\right) \times p\left(\mathbf{w}_{7} \mid \mathbf{w}_{5}, \mathbf{w}_{6}, \mathbf{y}\right) \\
& \times p\left(\mathbf{w}_{4} \mid \mathbf{w}_{5}, \mathbf{w}_{7}, \mathbf{y}\right) \times p\left(\mathbf{w}_{8} \mid \mathbf{w}_{6}, \mathbf{w}_{7}, \mathbf{y}\right)=p\left(\mathbf{w}_{1}, \mathbf{w}_{2}, \mathbf{w}_{3}, \mathbf{w}_{4}, \mathbf{w}_{5}, \mathbf{w}_{6}, \mathbf{w}_{7}, \mathbf{w}_{8} \mid \mathbf{y}\right)
\end{aligned}
$$

\subsubsection{Creating blocks by conditioning}

The method of random propagation of Dawid [3] can be used to obtain a random sample of all variables from their joint posterior distribution, $p(\mathbf{V} \mid \mathbf{e})$, or equivalently $p\left(\mathbf{w} \mid \mathbf{b}, \mathbf{u}, \mathbf{m}, f, \sigma_{\mathrm{e}}^{2}, \sigma_{\mathrm{u}}^{2}, \mathbf{y}\right)$. However, if the Bayesian network is large and complex, the cliques of the junction tree may contain many variables. This is a problematic scenario, as dimensions of the corresponding belief tables are exponential in the number of variables the cliques contain. Therefore, the storage requirements of junction trees may become prohibitive, preventing the performance of the operations described earlier. If this were the case, it would not be possible to obtain a random sample from the joint distribution of all variables.

Conditioning on a variable allows a new Bayesian network to be drawn, where the variable conditioned on is separated into several clones. This will often break loops in the network, as illustrated in figure 3. When loops are broken, fewer fill-in links are needed to render the graph triangulated, and consequently, fewer and smaller cliques are created. It follows that the storage requirements of the corresponding junction tree are smaller and random propagation can be performed. The concept of conditioning is illustrated in figure 3, where two different variables, $\mathbf{w}_{5}$ and $\mathbf{w}_{7}$, are conditioned on. The resulting Bayesian networks are illustrated in figure $3 a$ and $c$, and the reduced junction trees are illustrated in figure $3 b$ and $d$. This corresponds to the creation of two blocks, $B_{1}=\left\{\mathbf{w}_{1} \mathbf{w}_{2} \mathbf{w}_{3} \mathbf{w}_{4} \mathbf{w}_{6} \mathbf{w}_{7} \mathbf{w}_{8}\right\}$ and $B_{2}=\left\{\mathbf{w}_{1} \mathbf{w}_{2} \mathbf{w}_{3} \mathbf{w}_{4} \mathbf{w}_{5} \mathbf{w}_{6} \mathbf{w}_{8}\right\}$. The reduced junction trees demonstrate that storage requirements of the junction trees are reduced, because loops in the original Bayesian network are broken. This occurs as the junction trees contain fewer cliques and some of the cliques contain fewer unknown variables. In figure $3 b$ and $d$ variables with letter subscripts are assumed known. It is easy to see that a very large junction tree can be reduced sufficiently with respect to storage constraints, if many variables are conditioned on.

Blocks were created by choosing variables through the following iterative steps. First, the variable yielding the highest reduction in storage requirements when conditioned on was identified. Second, this variable was conditioned on in some blocks. Third, the storage requirements of the resulting junction trees were 


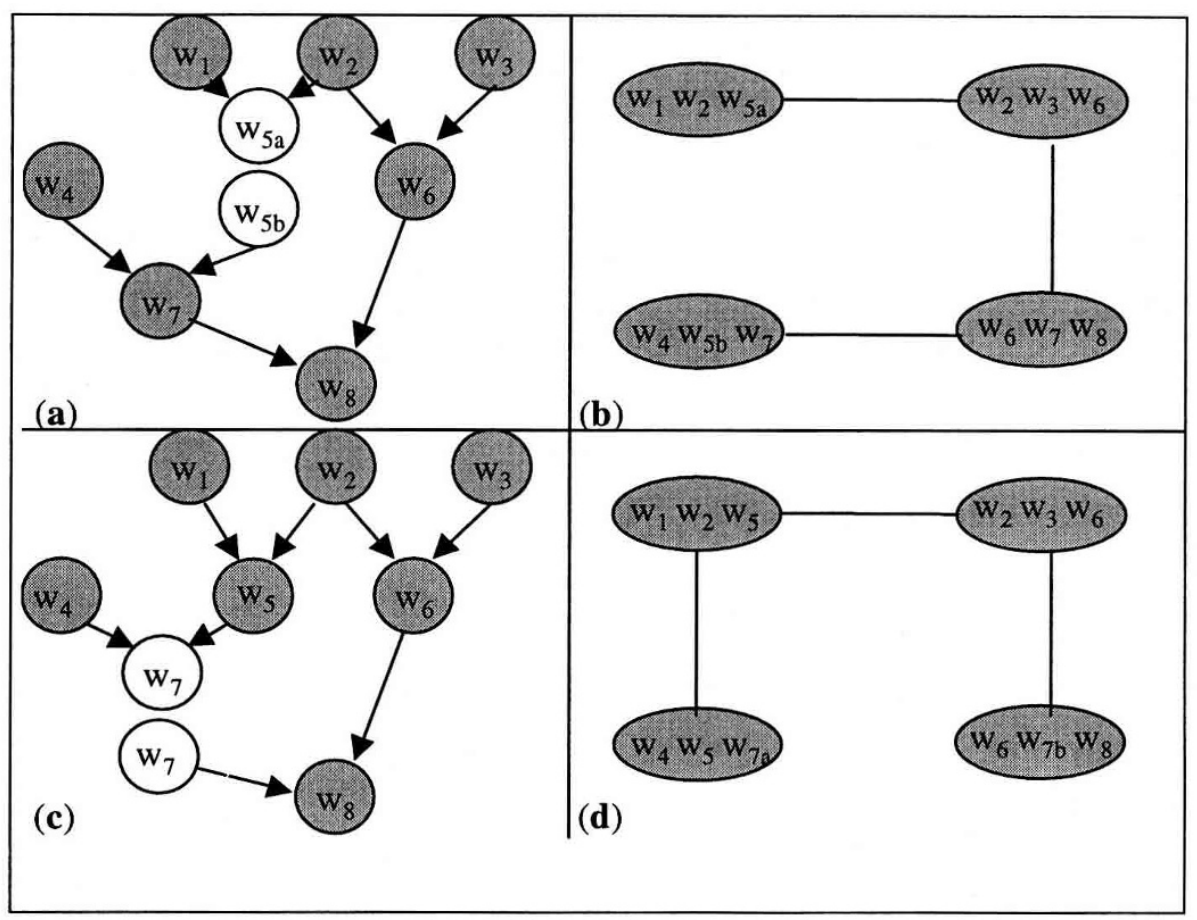

Figure 3. In the Bayesian network of figure $1 a \mathbf{w}_{5}$ is conditioned on in (a) and $\mathbf{w}_{7}$ in (c) enabling us to break the loop of the graph; (b) and (d) show the resulting junction trees.

calculated. If the storage requirements were larger than what was available, the steps were repeated finding the next variable to condition on. This was continued until all blocks satisfied the storage constraints of the system. All variables were, of course, required to be in at least one block. Jensen [10] provides more information on the block selection algorithm.

Blocks $B_{1}, \ldots, B_{\mathrm{n}}$ are constructed such that each contains most of the variables of the network. The complementary sets (i.e. the variables that are conditioned on) are called $B_{1}^{\mathrm{c}}, \ldots, B_{\mathrm{n}}^{\mathrm{c}}$. In this way, each set, $B_{\mathrm{i}} \cup B_{\mathrm{i}}^{\mathrm{c}}$, contains all the major genotypes in the pedigree. As the junction tree of each block can now be stored in the computer, exact inference can be performed, and a joint sample of all variables in the block can be obtained using the random propagation method. Therefore, using the described form of blocking, we can obtain random samples from the joint distribution of a block, conditional on the complementary set of other variables in the MIM and data, $p\left(B_{\mathrm{i}} \mid B_{\mathrm{i}}^{\mathrm{c}}, \mathbf{b}, \mathbf{u}, \mathbf{m}, \sigma_{\mathrm{e}}^{2}, \sigma_{\mathbf{u}}^{2}, f, \mathbf{y}\right)$.

In the MIM, the Gibbs sampling algorithm using general blocking can thus be summarised as follows:

1) form optimal blocks with respect to storage constraints by conditioning;

2) construct the junction tree for each block;

3) run the Gibbs sampler as shown previously, but substitute step II by: 
IIa) propagate information to $B_{\mathrm{i}}$, from new updates of allele frequency, adjusted phenotypes and genotypes of $B_{\mathrm{i}}^{\mathrm{c}}$ using the message passing scheme;

IIb) use random propagation of Dawid [3] to achieve a random sample from $p\left(B_{\mathrm{i}} \mid B_{\mathrm{i}}^{\mathrm{c}}, \mathbf{b}, \mathbf{u}, \mathbf{m}, \boldsymbol{\sigma}_{\mathrm{e}}^{2}, \sigma_{\mathrm{u}}^{2}, f, \mathbf{y}\right)$;

IIc) sample genotypes in $B_{\mathrm{i}}^{\mathrm{c}}$ according to equation (3).

Each time step II is performed a new block $B_{\mathbf{i}+1}$ is updated. When all blocks have been sampled we start again sampling $B_{1}$.

In this algorithm, only one of the blocks $\left(B_{\mathrm{i}}\right)$ is updated during each iteration. All other variables are updated from their full conditional distributions, such that all variables are sampled once during each iteration. Other updating schemes are also possible, and are described in the discussion.

\section{EXAMPLE ON SIMULATED DATA}

As an example, a simulated pedigree, with five overlapping generations of individuals, was analysed. In each generation five randomly selected sires were each mated to ten randomly selected dams, and each dam produced a litter of three offspring. Parents for the next generation were selected from all offspring and the parents in the current generation. This results in a pedigree of 750 individuals. Input values for the simulated data set were: $\sigma_{\mathrm{e}}^{2}=85, \sigma_{\mathrm{u}}^{2}=15$, $a=10, f=0.2$ and $\sigma_{\mathrm{mg}}^{2}=32$, where $\sigma_{\mathrm{mg}}^{2}$ is the expected major gene variance calculated as $\sigma_{\mathrm{mg}}^{2}=2 p(1-p) a^{2}$. In the simulation, as well as in the analysis, inbreeding was ignored.

The simulated data set was analysed using the general blocking algorithm with five blocks, each containing more than $95 \%$ of all major genotypes. The sampling scheme of Janss et al. [9] (sire blocking), was used as a reference method. The algorithm in which all variables are updated univariately from the full conditional distributions is not included in the present study because sire blocking has already been shown to be much more efficient [9].

Preliminary analysis showed that 100000 samples would be sufficient for the given Markov chain to obtain the equivalent of 250 independent samples of genetic variances. A burn-in of 5000 samples was used to allow the chain to converge to the target distribution.

\subsection{Convergence and mixing diagnostics}

The criteria upon which we compare the two algorithms is an assessment of the information content in a given Gibbs chain. The effective sample size measures the number of independent samples from the marginal posterior distribution to which the actual chain corresponds. To estimate the effective sample size, a standardised time series method of batch means was used $[6,7]$. This relies on dividing the chain, of length $n$, into $m$ equal-sized batches, with the batch means calculated as: $M_{\mathrm{k}}=\frac{m}{n} \sum_{i=(k-1) N / m+1}^{k n / m} g\left(X_{\mathrm{i}}\right)$, $k=1, \ldots, m$, and $X_{\mathbf{i}}$ are the samples. These batch means will converge in distribution to independent, identically distributed normal random variables. Convergence of the batch means was checked by standard one-way analysis 
of variance and by estimating the lag correlation between batch means. The Monte Carlo variance $\left(V_{\mathrm{MC}}\right)$ was estimated as the variance between batches: $V_{\mathrm{MC}}=\frac{1}{m(m-1)} \sum_{k=1}^{m}\left(M_{\mathrm{k}}-\bar{M}\right)^{2}, k=1, \ldots, m$, where $\bar{M}$ was the mean of the $m$ batch means, and the effective sample size [21]: $\mathrm{SS}_{\mathrm{E}}=V_{W} / V_{\mathrm{MC}}$, where $V_{W}$ was the within-batch variance, calculated using time series analysis, to take the high autocorrelation of successive samples into account.

\subsection{Results from simulated example}

The marginal posterior mean of the residual variance is low compared to the input parameters of the simulated data set (tables $I$ and $I I$ ). It is not a general feature of the algorithm to underestimate the residual variance, and the input values of parameters were all contained in the $95 \%$ highest posterior density regions estimated for the relevant marginal posterior densities.

Table I. Posterior mean and standard deviation (SD) of parameters in the model, with their Monte Carlo variance (MCV) and effective sample size $\left(\mathrm{SS}_{\mathrm{E}}\right)$ using general blocking for 100000 rounds.

\begin{tabular}{lllll}
\hline Parameter & Mean & SD & MCV & SS $_{\mathrm{E}}$ \\
\hline$\sigma_{\mathrm{e}}^{2}$ & 75.50 & 7.62 & 0.064 & 833 \\
$\sigma_{\mathrm{u}}^{2}$ & 13.7 & 11.59 & 0.56 & 225 \\
$\sigma_{\mathrm{mg}}^{2}$ & 39.27 & 12.51 & 0.61 & 400 \\
$|a|$ & 11.12 & 2.00 & 0.015 & 240 \\
$f$ & 0.2149 & 0.1046 & 0.000026 & 390 \\
\hline
\end{tabular}

Table II. Posterior mean and standard deviation (SD) of parameters in the model, with their Monte Carlo variance $(\mathrm{MCV})$ and effective sample $\left(\mathrm{SS}_{\mathrm{E}}\right)$ size using sire blocking for 100000 rounds.

\begin{tabular}{llllc}
\hline Parameter & Mean & SD & MCV & SS $_{\mathrm{E}}$ \\
\hline$\sigma_{\mathrm{e}}^{2}$ & 75.40 & 7.85 & 0.15 & 378 \\
$\sigma_{\mathrm{u}}^{2}$ & 17.19 & 13.6 & 3.74 & 46 \\
$\sigma_{\mathrm{mg}}^{2}$ & 36.4 & 14.27 & 3.30 & 58 \\
$|a|$ & 11.43 & 3.41 & 0.057 & 171 \\
$f$ & 0.1929 & 0.1066 & 0.00008 & 126 \\
\hline
\end{tabular}

Estimated means of marginal posterior distributions were very similar for the two Gibbs sampling approaches. However, important differences in mixing properties, measured as Monte Carlo variance and effective sample size, were observed. The Monte Carlo variance was considerably larger and effective 
sample sizes considerably smaller when using the sire blocking algorithm (table $I I$ ), compared to the general blocking strategy (table $I$ ). For example, for the parameter of major gene variance, which was of primary interest, the $\mathrm{SS}_{\mathrm{E}}$ was nearly seven times as high when the general blocking strategy was used. Therefore, the sire blocking scheme was allowed to run another 100000 rounds and the results are summarised in table III. The effective sample sizes of the two main parameters of genetic variance $\left(\sigma_{\mathrm{u}}^{2}\right.$ and $\left.\sigma_{\mathrm{mg}}^{2}\right)$ were still considerably smaller than when the general blocking algorithm was run for half as many rounds. For example, the $\mathrm{SS}_{\mathrm{E}}$ for the major gene variance was still nearly four times as high when using the general blocking algorithm. These results indicate that, even when many final offspring exist, the general blocking algorithm mixes faster.

Table III. Posterior mean and standard deviation (SD) of parameters in the model, with their Monte Carlo variance (MCV) and effective sample size $\left(\mathrm{SS}_{\mathrm{E}}\right)$ using sire blocking for 200000 rounds.

\begin{tabular}{llllc}
\hline Parameter & Mean & SD & MCV & SS $_{\mathbf{E}}$ \\
\hline$\sigma_{\mathrm{e}}^{2}$ & 75.30 & 7.76 & 0.048 & 1161 \\
$\sigma_{\mathrm{u}}^{2}$ & 15.29 & 11.96 & 1.64 & 81 \\
$\sigma_{\mathrm{mg}}^{2}$ & 38.23 & 12.91 & 1.47 & 106 \\
$|a|$ & 11.30 & 3.09 & 0.013 & 510 \\
$f$ & 0.2046 & 0.0968 & 0.00003 & 222 \\
\hline
\end{tabular}

The algorithms were primarily compared with respect to the number of rounds, instead of computer time. This was because the general relationship between number of rounds and computer time for the two algorithms in different data structures was not known. However, in terms of computer time, one round of general blocking corresponds to almost two rounds using sire blocking. Consequently, when comparing the usage of computer time by the different algorithms, tables $I$ and $I I I$ can be compared directly.

\section{DISCUSSION}

The primary objective of this paper was to introduce graph theoretic methods to animal breeding, with particular emphasis on improving mixing and reducibility properties in MCMC methods for single gene models. By introducing a blocking Gibbs sampler with the MIM in a segregation analysis setting, the blocking algorithm of Jensen et al. [13] was extended to methods used in quantitative genetics. However, if genetic marker information is included in the model, more severe reducibility problems are often encountered, making a Gibbs sampler with univariate updating infeasible. This is because the sample space is often cut into non-communicating subspaces, and the induced Markov chain does not converge to the desired joint posterior distribution. However, sampling strategic individuals jointly will connect the disjoint sample spaces, and thereby create an irreducible Gibbs sampler. Blocking Gibbs sampling has 
already been successfully applied to linkage analysis with one genetic marker for a simple Mendelian trait [11]. This approach can also be extended to complex models such as the MIM. Although in a complex pedigree, it might not be obvious which genotypes must be sampled jointly, the general blocking strategy holds the potential to solve the crucial reducibility problem in MCMC methods for linkage analysis.

The two blocking strategies resulted in similar point estimates of marginal posterior means of model parameters. However, in this simulated example, the general blocking strategy was more efficient. After having run the algorithms for an equal number of iterations, the samples from the general blocking algorithm contained approximately seven times as much information concerning the major gene variance, as did the samples from the sire blocking algorithm (measured by the effective sample sizes). When the algorithms were run for the same amount of time, rather than the same number of iterations, the samples from the general blocking algorithm still contained four times as much information as those from the sire blocking algorithm. The difference in efficiency might seem small, but for these time-consuming algorithms, it is quite a significant difference.

The simulated data set used to compare the two blocking strategies had rather many final offspring. This meant that sire blocking was already a considerable improvement in comparison to using univariate updating of genotypes. Therefore, a large difference between the two blocking strategies was not expected. In other situations, the proposed algorithm is expected to be even more beneficial. Examples include a pedigree with fewer final offspring or with many individuals without phenotypic records. Such cases often occur when conducting analyses in animal breeding, as ancestors are often traced back several generations from individuals with phenotypic records. The result is a complex pedigree with a gap between founder individuals and individuals having records, but not much information on the genotypes. In such situations, the general blocking strategy is also expected to improve mixing considerably because founder individuals will be sampled jointly with those having records.

In the proposed algorithm, blocks are chosen to be as large as possible in order to jointly sample almost all genotypes. Thus, it was chosen to update one block of genotypes in each iteration. The drawback of this approach is that it is currently difficult to handle a complex pedigree of more than about 5000 to 10000 individuals. However, this particular strategy is not essential. The use of the graphical model theory to construct a blocking Gibbs sampler is much more general and can be applied in many different ways. A block selection algorithm that ensures the possibility of using the general blocking strategy, for any sized pedigree, could be developed. In such an algorithm, each block will, in large data sets, contain a much smaller proportion of the entire pedigree and possibly with less overlap between the blocks. Consequently, the scheme in which the blocks are visited also has to be revised to optimise the algorithm. In this situation several blocks would be updated in each iteration, but not necessarily all. In order to analyse large animal pedigrees, development of such blocking schemes is required. 


\section{REFERENCES}

[1] Bulmer M.G., The effect of selection on genetic variability, Am. Nat. 105 (1971) 201-211.

[2] Cannings C., Thompson E.A., Skolnick M.H., Probability functions on complex pedigrees, Adv. Appl. Probab. 10 (1978) 26-61.

[3] Dawid A.P., Applications of a general propagation algorithm for probabilistic expert systems, Stat. Comput. 2 (1992) 25-36.

[4] Elston R.C., Stewart J., A general model for genetic analysis of pedigree data, Hum. Hered. 2 (1971) 523-542.

[5] Gelfand A.E., Smith A.F.M., Sampling based approaches to calculating marginal densities, J. Am. Assoc. 85 (1990) 398-409. 511

[6] Geyer C.J., Practical Markov chain Monte Carlo, Stat. Sci. 7 (4) (1992) 473-

[7] Glynn P.W., Inglehart D.L., Simulation output analysis using standardised time series, Math. Oper. Res. 15 (1990) 1-16.

[8] Guo S.W., Thompson E.A., A Monte Carlo method for combined segregation and linkage analysis, Am. J. Hum. Genet. 51 (1992) 1111- 1126.

[9] Janss L.L.G., Thompson R., Van Arendonk J.A.M., Application of Gibbs sampling for inference in a mixed major gene-polygenic inheritance model in animal populations, Theor. Appl. Genet. 91 (6/7) (1995) 1137-1147.

[10] Jensen C.S., Blocking Gibbs sampling for inference in large and complex Bayesian networks with application in genetics, Ph.D. thesis, Aalborg University, Denmark, 1997.

[11] Jensen C.S., Kong A., Blocking Gibbs sampling for linkage analysis in large pedigrees with many loops, Technical report R-96-2048, Department of Computer Science, Aalborg University, Denmark, 1996.

[12] Jensen C.S., Sheehan N., Problems with the determination of the noncommunicating classes for MCMC applications in Pedigree Analysis, Biometry 54 (1997) 416-425.

[13] Jensen C.S., Kong A., Kjærulff U., Blocking-Gibbs sampling in very large probabilistic expert systems, Int. J. Hum. Comp. Stud. 42 (1995) 647-666.

[14] Jensen F.V., An Introduction to Bayesian Networks, UCL Press, University College Limited, CA, 1996.

[15] Jensen F.V., Lauritzen S.L., Olesen K.G., Bayesian updating in causal probabilistic networks by local computations, Comput. Stat. Q. 4 (1990) 269-282.

[16] Lauritzen S.L., Spiegelhalter D.J., Local computations with probabilities on graphical structures and their application to expert systems, J. R. Stat. Soc. Series B 50 (1988) 157-224.

[17] Lindley D.V., Smith A.F.L., Bayes estimates for the linear model, J. R. Stat. Soc. Series B 34 (1972) 1-41. 1976.

[18] Morrison D.F., Multivariate Statistical Methods, McGraw-Hill, New York,

[19] Morton N.E., MacLean C.J., Analysis of family resemblance. III. Complex segregation analysis of quantitative traits, Am. J. Hum. Genet. 26 (1974) 489-503.

[20] Sheehan N., Genetic restoration on complex pedigrees, $\mathrm{Ph} . \mathrm{D}$. thesis, University of Washington, 1990.

[21] Sorensen D.A., Andersen S., Gianola D., Korsgaard I., Bayesian inference in threshold models using Gibbs sampling, Genet. Sel. Evol. 27 (1995) 229-249.

[22] Wang C.S., Rutledge J.J., Gianola D., Marginal inference about variance components in a mixed linear model using Gibbs sampling, Genet. Sel. Evol. 25 (1993) 41-62. 